\title{
On Ramsey Minimal Graphs for a 3-Matching Versus a Path on Five Vertices
}

\author{
Kristiana Wijaya $^{1, *}$, Edy Tri Baskoro ${ }^{2}$, Asep Iqbal Taufik ${ }^{3}$, Denny Riama Silaban ${ }^{3}$
}

\author{
${ }^{I}$ Graph, Combinatorics, and Algebra Research Group, Department of Mathematics, FMIPA, Universitas Jember \\ ${ }^{2}$ Combinatorial Mathematics Research Group, FMIPA, Institut Teknologi Bandung \\ ${ }^{3}$ Department of Mathematics, Faculty of Mathematics and Natural Sciences, Universitas Indonesia, Depok 16424 \\ *Corresponding author. Email: kristiana.fmipa@unej.ac.id
}

\begin{abstract}
Let $G, H$, and $F$ be simple graphs. The notation $F \rightarrow(G, H)$ means that any red-blue coloring of all edges of $F$ contains a red copy of $G$ or a blue copy of $H$. The graph $F$ satisfying this property is called a Ramsey $(G, H)$-graph. A Ramsey $(G, H)$-graph is called minimal if for each edge $e \in E(F)$, there exists a red-blue coloring of $F-e$ such that $F-e$ contains neither a red copy of $G$ nor a blue copy of $H$. In this paper, we construct some Ramsey $\left(3 K_{2}, P_{5}\right)$-minimal graphs by subdivision ( 5 times) of one cycle edge of a Ramsey $\left(2 K_{2}, P_{5}\right)$-minimal graph. Next, we also prove that for any integer $m \geq 3$, the set $R\left(m K_{2}, P_{5}\right)$ contains no connected graphs with circumference 3 .
\end{abstract}

Keywords: Ramsey minimal graph, 3-matching, Path.

\section{INTRODUCTION}

Given simple graphs $G$ and $H$, any red-blue coloring of the edges of $F$ is called a $(G, H)$-coloring if it has neither red copy of $G$ nor blue copy of $H$. The notation $F \rightarrow(G, H)$ means that in any red-blue coloring of $F$ there exists a red copy of $G$ or a blue copy of $H$ as a subgraph. A graph $F$ is said to be a Ramsey $(G, H)$ minimal if $F \rightarrow(G, H)$ but for any $e \in E(F)$ there exists a $(G, H)$-coloring on graph $F-e$. The set of all Ramsey $(G, H)$-minimal graphs is denoted by $R(G, H)$. Burr, Erdős, Faudree, and Schelp [1] proved that if $H$ is an arbritary graph then $R\left(m K_{2}, H\right)$ is a finite set. One of challenging problems in Ramsey Theory is to characterize all graphs in the set $R\left(m K_{2}, H\right)$ for a given graph $H$. As usual, $K_{n}, C_{n}$, and $P_{n}$ denote a complete graph, a cycle, and a path on $n$ vertices, respectively. For any connected graph $G$, and $m \geq 2$, the notation $m G$ means a disjoint union of $m$ copies of a graph $G$. A $t$ matching, denoted by $t K_{2}$, is a graph with $t$ components where every component is a graph $K_{2}$.

In general, it is difficult to characterize all graphs belonging to $R\left(m K_{2}, H\right)$. However, for some particular graph $H$, this set $R\left(m K_{2}, H\right)$ has been known. For instance, Burr, Erdős, Faudree, and Schelp [1] showed that $R\left(2 K_{2}, 2 K_{2}\right)=\left\{C_{5}, 3 K_{2}\right\}$ and $R\left(2 K_{2}, K_{3}\right)=$ $\left\{K_{5}, 2 K_{3}, G_{1}\right\}$, where $G_{1}$ is a graph having the vertex-set
$V\left(G_{1}\right)=\left\{c, u_{i}, v_{i}, w_{i} \mid i=1,2\right\}$ and the edge-set $E\left(G_{1}\right)$ $=\left\{c u_{i}, c v_{i}, c w_{i} \mid i=1,2\right\} \cup\left\{u_{1} u_{2}, v_{1} v_{2}, w_{1} w_{2}\right\} \cup$ $\left\{u_{1} v_{1}, u_{1} w_{1}, v_{1} w_{1}\right\}$. Burr et al. [2] showed that $R\left(2 K_{2}, P_{3}\right)=\left\{C_{4}, C_{5}, 2 P_{3}\right\}$. Baskoro and Yulianti [3] proved that $R\left(2 K_{2}, P_{4}\right)=\left\{C_{5}, C_{6}, C_{7}, 2 P_{4}, C_{4}^{+}\right\}$, where $C_{4}^{+}$ is a graph formed by a cycle on 4 vertices $C_{4}$ and two pendants vertices so that two vertices of degree 3 in the cycle $C_{4}$ are adjacent. Furthermore, they [3] also proved that $R\left(2 K_{2}, P_{5}\right)=\left\{C_{6}, C_{7}, C_{8}, C_{9}, 2 P_{5}\right\} \cup\left\{A_{i} \mid i \in[1,7]\right\}$, where $A_{i} \mathrm{~s}$ are the graphs depicted in Figure 1. Wijaya, Baskoro, Assiyatun, and Suprijanto [4] showed that the cycle $C_{s}$ belongs to $R\left(m K_{2}, P_{n}\right)$ if and only if $s \in[m n-$ $n+1 \leq s \leq m n-1]$. Other results on characterizing all Ramsey minimal graphs for the pair of a matching versus a path can be seen in $[5-8]$.

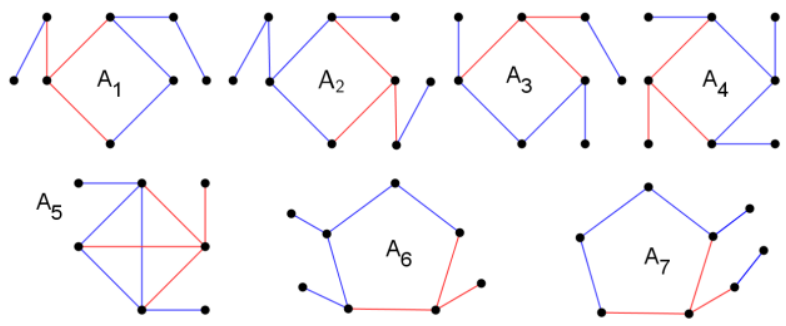

Figure 1 Some Ramsey $\left(2 K_{2}, P_{5}\right)$-minimal graphs. 
In [1], Burr, Erdős, Faudree, and Schelp gave a family of $\frac{(n+1)}{2}$ non-isomorphic graphs in $R\left(2 K_{2}, K_{n}\right)$ for $n \geq 4$. These graphs are constructed from a complete graph $K_{n+1}$. In the same paper, Burr, Erdös, Faudree, and Schelp also gave a family of $(n-2)$ non-isomorphic graphs belonging to $R\left(2 K_{2}, K_{1, n}\right)$. Motivated by them, Wijaya, Baskoro, Assiyatun, and Suprijanto [9] constructed some graphs in $R\left(m K_{2}, P_{3}\right)$ by subdivision (3 times) on any non-pendant edge of a connected graph in $R\left((m-1) K_{2}, P_{3}\right)$. Furthermore, Wijaya, Baskoro, Assiyatun, and Suprijanto [10] constructed a family of Ramsey $\left(m K_{2}, P_{4}\right)$ minimal graphs from any Ramsey $\left((m-1) K_{2}, P_{4}\right)$ minimal graph by the subdivision process on any cycle-edge (4 times).

In this paper, we focus on constructing Ramsey $\left(3 K_{2}, P_{5}\right)$ minimal graphs for 3-matching versus a path with five vertices. We also prove that there is no graph with circumference 3 belonging to $R\left(m K_{2}, P_{5}\right)$ for any integer $m \geq 3$. A circumference of a graph is the length of the longest cycle in that graph.

The following two lemmas provide the necessary and sufficient conditions for any graph in $R\left(3 K_{2}, H\right)$ for any graph $H$.

Lemma 1.1 $[9,10]$ For any fixed graph $H$, the graph $F \rightarrow\left(3 K_{2}, H\right)$ holds if and only if the following four conditions are satisfied: (i) $F-\{u, v\} \supseteq H$ for each $u, v \in V(F)$, (ii) $F-\{u\}-E\left(K_{3}\right) \supseteq H$ for each $u \in$ $V(F)$ and a triangle $K_{3}$ in $F$, (iii) $F-E\left(2 K_{3}\right) \supseteq H$ for every two triangles in $F$, (iv) $F-E\left(S_{5}\right) \supseteq H$ for every induced subgraph with 5 vertices $S$ in $F$.

Lemma 1.2 $[9,10]$ Let $H$ be a simple graph. Suppose $F$ is a Ramsey $\left(3 K_{2}, H\right)$-graph. $F$ is said to be minimal if for each $e \in E(F)$ satisfies $(F-e) \nrightarrow\left(3 K_{2}, H\right)$, that is, (i) $(F-e)-\{u, v\} \nsupseteq H$ for each $u, v \in V(F)$, (ii) $F-$ $\{u\}-E\left(K_{3}\right) \nsupseteq H$ for each $u \in V(F)$ and a triangle $K_{3}$ in $F$, (iii) $F-E\left(2 K_{3}\right) \nsupseteq H$ for every two triangles in $F$, (iv) $F-E\left(S_{5}\right) \nsupseteq H$ for every induced subgraph with 5 vertices $S$ in $F$.

Any graph satisfying all conditions stated in Lemmas 1 and 2 is a Ramsey $\left(3 K_{2}, H\right)$-minimal graph. The condition stated in Lemma 1.2 is called the minimality property of a graph in $R\left(3 K_{2}, H\right)$.

Next theorem is one of the important properties of a Ramsey $\left(m K_{2}, H\right)$-minimal graph.

Theorem 1.3 [9] Let $H$ be a graph and $m>1$ be an integer. If $F \in\left(m K_{2}, H\right)$, then for any $v \in V(F)$ and $K_{3} \subseteq F$, both graphs $F-\{v\}$ and $F-E\left(K_{3}\right)$ contain a
Ramsey $\left((m-1) K_{2}, H\right)$-minimal graph.

\section{MAIN RESULTS}

In this section, we give some graphs belonging to $R\left(3 K_{2}, P_{5}\right)$. We construct these graphs by the subdivision process on any cycle edge of a connected graph in $R\left(2 K_{2}, P_{5}\right)$ depicted in Figure 1. Before doing this, first we show that a graph $F_{1}$, depicted in Figure 2 , is a Ramsey $\left(3 K_{2}, P_{5}\right)$-minimal graph. The vertex set of a graph $F_{1}$ is $V\left(F_{1}\right)=\left\{v_{1}, v_{2}, \ldots, v_{11}\right\}$ and the edge set of a graph $F_{1}$ is $E\left(F_{1}\right)=\left\{v_{i} v_{i+1} \mid i=1,2, \ldots, 10\right\} \quad U$ $\left\{v_{2} v_{9}, v_{3} v_{10}\right\}$.

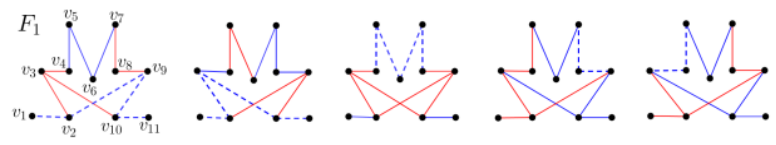

Figure 2 A graph $F_{1}$ and some red-blue colorings of $F_{1}$ so that $F_{1}$ contains no red $3 K_{2}$ but it contains a blue $P_{5}$.

Proposition 2.1 Let $F_{1}$ be a graph on 11 vertices and 12 edges as depicted in Figure 2. The graph $F_{1}$ is a Ramsey $\left(3 K_{2}, P_{5}\right)$-minimal graph.

Proof. First, we prove that for any red-blue coloring of $F_{1}$ there exists a red $3 K_{2}$ or a blue $P_{5}$ in $F_{1}$. We can see that $F_{1}-\left\{v_{i}, v_{j}\right\}$ always contains a path $P_{5}$ for any $1 \leq$ $i, j \leq 11$. It can be verified that $F_{1}-E\left(S_{5}\right) \supseteq H$ for every induced subgraph with 5 vertices $S$ in $F_{1}$. Since $F_{1}$ has no triangle then by Lemma $1.1, F_{1} \rightarrow\left(3 K_{2}, P_{5}\right)$. Next, we prove the minimality property of $F_{1}$. For any edge $e$ we will show that $\left(F_{1}-e\right) \nrightarrow\left(3 K_{2}, P_{5}\right)$. If $e$ is one of dashed edges in Figure 2, then each red-blue coloring in Figure 2 provides a $\left(3 K_{2}, P_{5}\right)$ coloring on $F_{1}-e$, namely a coloring that have neither red $3 K_{2}$ nor blue $P_{5}$. Therefore $F_{1} \in R\left(3 K_{2}, P_{5}\right)$.

Next, we construct some Ramsey $\left(3 K_{2}, P_{5}\right)$-minimal graphs from previous known Ramsey $\left(2 K_{2}, P_{5}\right)$-minimal graphs by subdivision process. Consider each of Ramsey $\left(2 K_{2}, P_{5}\right)$-minimal graphs in Figure 1 . By the subdivision (5 times) on any of its cycle-edges we produce Ramsey $\left(3 K_{2}, P_{5}\right)$-minimal graphs in Figure 3 . In total, we obtain 12 non-isomorphic graphs belonging to $R\left(3 K_{2}, P_{5}\right)$. Two non-isomorphic graphs $F_{2}$ and $F_{3}$ are obtained from the subdivision of $A_{1}$. Two non-isomorphic graphs $F_{4}$ and $F_{5}$ are formed from $A_{2}$. Two non-isomorphic graphs $F_{6}$ and $F_{7}$ are obtained from $A_{3}$. One graph called $F_{8}$ is obtained from the graph $A_{4}$. One graph $F_{9}$ is formed from $A_{5}$. Two non-isomorphic graphs $F_{10}$ and $F_{11}$ are obtained from the graph $A_{6}$. Last, two non-isomorphic graphs $F_{12}$ and $F_{13}$ are formed from $A_{7}$. In the following theorem, we will 
prove that these graphs are Ramsey $\left(3 K_{2}, P_{5}\right)$-minimal graphs.

Theorem 2.2 All the graphs $F_{2}, F_{3}, \ldots, F_{13}$ in Figure 3 are Ramsey $\left(3 K_{2}, P_{5}\right)$-minimal graphs.
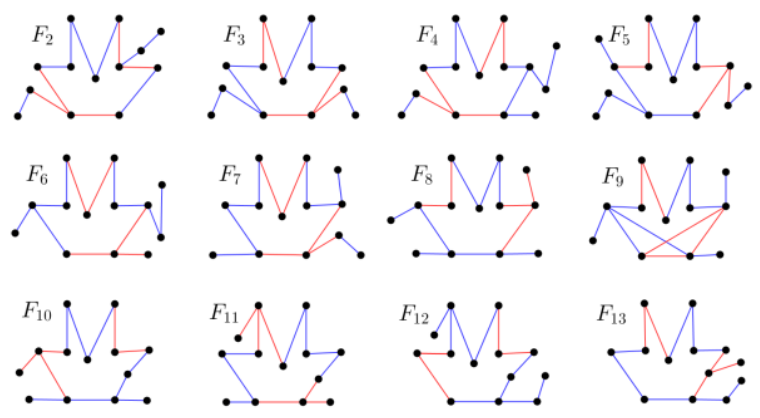

Figure 3 Some graphs belong to $R\left(3 K_{2}, P_{5}\right)$.

Proof. Let $F$ be any graph in Figure 3. It is easy to see that $F$ satisfies all the conditions in Lemma 1.1. Then, $F \rightarrow\left(3 K_{2}, P_{5}\right)$ holds. Now, we will show the minimality property of $F$. Let $e$ be any edge in $F$. If $e$ is one of dashed edges, then a $\left(3 K_{2}, P_{5}\right)$-coloring on $F-e$ is provided in Figures 4 and 5 for all cases of $F$ and $e$.
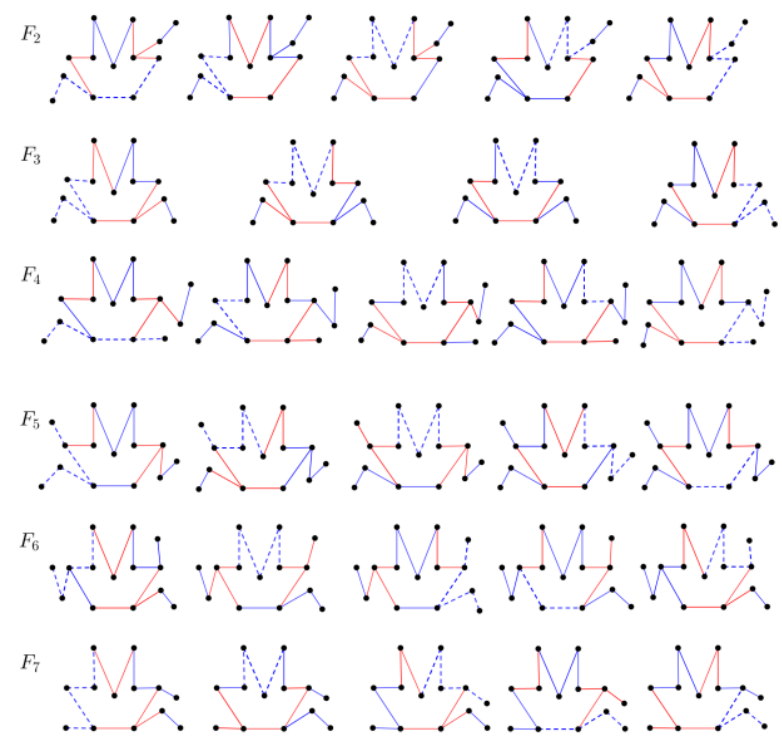

Figure 4 The $\left(3 K_{2}, P_{5}\right)$-colorings on $F_{i}-e$ if $e$ is one of dashed edges and for $i \in[2,7]$.

Actually, there are two non-isomorphic graphs obtained by the subdivision (5 vertices) on any cycle edge of $A_{5}$ (see Figure 1). One of these two graphs is $F_{10}$ and the other is obtained by subdivision ( 5 vertices) on the edge incident with a vertex of degree 4 and a vertex of degree 3 . The last graph is a Ramsey- $\left(3 K_{2}, P_{5}\right)$ graph but not minimal since it contains a graph $F_{1} \in R\left(3 K_{2}, P_{5}\right)$ (in Figure 2).

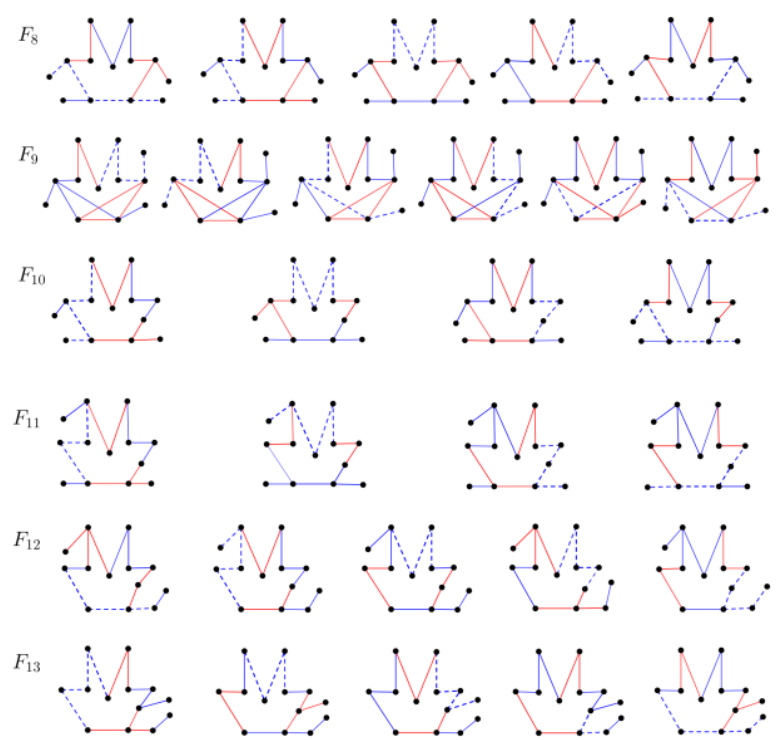

Figure 5 The $\left(3 K_{2}, P_{5}\right)$-colorings on $F_{i}-e$ for $i \in$ $[8,13]$ if $e$ is one of dashed edges.

In the following theorem, we will give a property of graphs belonging to $R\left(m K_{2}, P_{5}\right)$.

Theorem 2.3 There is no Ramsey $\left(m K_{2}, P_{5}\right)$-minimal graph with circumference 3 for any integer $m \geq 2$.

Proof. We will prove the theorem by induction on $m$. If $m=2$ then it has been shown that there is no $\left(2 K_{2}, P_{5}\right)$ minimal graph with circumference 3 (see [3]).

Assume that there is no $\left(t K_{2}, P_{5}\right)$-minimal graph with circumference 3 for any positive integer $t \leq m-1$. We will show that there is no $\left(m K_{2}, P_{5}\right)$-minimal graph with circumference 3 . Suppose to the contrary that there exists a graph $F$ which is a Ramsey $\left(m K_{2}, P_{5}\right)$-minimal graph with circumference 3 . Then, $F$ must be a unicyclic graph. Let $C$ be the cycle in $F$ with $V(C)=\left\{u_{1}, u_{2}, u_{3}\right\}$. According to Theorem 1.3, $F-\left\{u_{i}\right\}$ for every $i \in[1,3]$ contains a graph $G \in R\left((m-1) K_{2}, P_{5}\right)$. By assumption, the set $R\left((m-1) K_{2}, P_{5}\right)$ has no graph with circumference 3. So, $G$ must be isomorphic to $(m-$ 1) $P_{5}$. It forces that $F-E(C)$ is a graph $P_{n_{1}} \cup P_{n_{2}} \cup P_{n_{3}}$ where $n_{1}+n_{2}+n_{3} \geq 5 m=15$. It implies that $F$ contains a graph $m P_{5}$. Hence, $F$ is not minimal. Otherwise, without loss of generality, we consider $n_{1}+$ $n_{2}+n_{3}=5 m-1 \geq 14$ and assume $u_{1} \in V\left(P_{n_{1}}\right), u_{2} \in$ $V\left(P_{n_{2}}\right)$, and $u_{3} \in V\left(P_{n_{3}}\right)$. Suppose w.l.o.g. $n_{1} \geq n_{2} \geq$ $n_{3}$ and $V\left(P_{n_{1}}\right)=\left\{u_{1}, v_{n_{1}-1}, v_{n_{1}-2}, \ldots, v_{2}, v_{1}\right\}$ where $v_{1}$ is the pendant vertex of a path $P_{n_{1}}$ and $E\left(P_{n_{1}}\right)=$ 
$\left\{u_{1} v_{n_{1}-1}, v_{i} v_{i+1} \mid i \in\left[1, n_{1}-2\right]\right\}$. Clearly $n_{1} \geq 5$. If $n_{1}>5$, we set the vertex $v_{5} \in V\left(P_{n_{1}}\right)$, then we obtain that $F-\left\{v_{5}\right\}$ does not contain a graph $(m-1) P_{5}$, which would contradict Theorem 1.3. In the case of $n_{1}=5$ we have $n_{2}=5$ and $n_{3}=4$. We obtain $F-\left\{u_{1}\right\} \nsupseteq 2 P_{5}$, a contradiction with Theorem 1.3. Thus, the proof is complete.

\section{CONCLUSION}

In this paper, we discuss on the construction of Ramsey $\left(3 K_{2}, P_{5}\right)$-minimal graphs. By the subdivision of any cycle edge of 7 Ramsey $\left(2 K_{2}, P_{5}\right)$-minimal graphs (in Figure 1) we obtain 13 non-isomorphic Ramsey $\left(3 K_{2}, P_{5}\right)$-minimal graphs. We also show that there is no Ramsey $\left(m K_{2}, P_{5}\right)$-minimal graph circumference 3 for any integer $m \geq 2$.

For a future work, we pose some open problems below.

Open Problem 1. Characterize all graphs belonging to $R\left(3 K_{2}, P_{5}\right)$ by excluding all graphs resulted in this paper.

Open Problem 2. Are there any connected graphs with circumference 4 or 5 belonging to $R\left(3 K_{2}, P_{5}\right)$ ?

Open Problem 3. Is it true that the subdivision (5 times) on any cycle-edge of a connected Ramsey ( $(m-$ 1) $\left.K_{2}, P_{5}\right)$-minimal graph always produces a connected Ramsey $\left(m K_{2}, P_{5}\right)$ - minimal graph?

\section{ACKNOWLEDGMENTS}

The second author thanks to the World Class Research (WCR) Program, Ministry of Research and Technology/National Research and Innovation Agency, Indonesia.

\section{REFERENCES}

[1] S.A. Burr, P. Erdős, R.J. Faudree, R.H. Schelp, A class of Ramsey-finite graphs, Proc. $9^{\text {th }}$ Conf. Combinatorics, Graph Theory, and Computing, pp. 171-180.
[2] S.A. Burr, P. Erdős, R.J. Faudree, C.C. Rousseau, R.H. Schelp, Ramsey-minimal graphs for matchings, Proceedings-Fourth International Graph Theory Conference, pp. 159-168.

[3] E.T. Baskoro and L. Yulianti, Ramsey minimal graphs for $2 K_{2}$ versus $P_{n}$, Advances and Applications in Discrete Mathematics 8, 2011, pp. 83-90. DOI: http://www.pphmj.com/journals/ articles/880.htm

[4] K. Wijaya, E.T. Baskoro, H. Assiyatun, D. Suprijanto, On unicyclic Ramsey $\left(m K_{2}, P_{3}\right)$ minimal graphs, Procedia Computer Science 74, 2015, pp. 10-14. DOI: https://doi.org/10.1016/j.procs.2015.12.067

[5] I. Mengersen, J. Oeckermann, Ramsey sets for matchings, Ars Combinatoria, 56, 2000, pp. 33-42.

[6] D. Tatanto and E.T. Baskoro, On Ramsey $\left(2 K_{2}, 2 P_{n}\right)$-minimal graphs, AIP Conference Proceedings 1450, 2012, 90. DOI: https://doi.org/10.1063/1.4724122

[7] H. Muhshi and E.T. Baskoro, On Ramsey $\left(3 K_{2}, P_{3}\right)$-minimal graphs, AIP Conference Proceedings 1450, 2012, $110 . \quad$ DOI: https://doi.org/10.1063/1.4724125

[8] K. Wijaya, E.T. Baskoro, H. Assiyatun, D. Suprijanto, On Ramsey $\left(4 K_{2}, P_{3}\right)$-minimal graphs, AKCE International Journal of Graphs and Combinatorics 15, 2018, pp. 174-186. DOI: https://doi.org/10.1016/j.akcej.2017.08.003

[9] K. Wijaya, E.T. Baskoro, H. Assiyatun, D. Suprijanto, On Ramsey $\left(m K_{2}, H\right)$-minimal graphs, Graphs and Combinatorics 33, 2017, pp. 233-243. DOI: https://doi.org/10.1007/s00373-016-1748

[10] K. Wijaya, E.T. Baskoro, H. Assiyatun, D. Suprijanto, Subdivision of graphs in $R\left(m K_{2}, P_{4}\right)$, Heliyon 6, 2020, e03843. DOI: https://doi.org/10.1016/j.heliyon.2020.e03843 